Lad. D u da: Beiträge z. Kenntniss d. Hemipteren-Fauna Böhmens. 169

\title{
Beiträge zur Kenntniss der Hemipteren-Fauna Böhmens.
}

Von Lad. Duda, k. k. Gymnasial-Professor in Königgrätz (Böhmen).

(4. Fortsetzung.)

\section{Lygaeosoma Spin.}

L. reticulatum H.-Sch. Mir bisher nur aus der Umgegend von Prag bekannt ( $\mathrm{Chvatěr} \mathrm{u} \mathrm{b),} \mathrm{im} \mathrm{Frühjahre} \mathrm{unter} \mathrm{Steinen}$ und trockenem Laub (mit der vorigen Art) gesammelt.

\section{Arocatus Spin. (Tetralaccus Fieb.)}

A. Roeselii Schum. An sonnigen Waldrändern, in der Rinde alter Nadelbäume, auch auf Erlen und anderem Gebüsch; in der Umgebung von Prag, selten (Krě, Zavist), auch von $\mathrm{Prach}$ notirt. Ein Exemplar habe ich auch von Hohenmauth.

\section{Nysius Dall.}

N. jacobeae Schill. In Holzschlägen und an Waldrändern, auf und unter Erdbeeren, wahrscheinlich nur in Gebirgsgegenden; mit Sicherheit kenne ich diese Art nur aus Johannisbad (im Riesengebirge), wo ich sie Ende 7 gesammelt (brachypt. Ex.) und aus Neuhaus, wo ich dieselbe am 27. April von einem blühenden Pflaumenbaume geklopft habe. In meiner böhm. Programmarbeit sind aus Versehen unrichtige Fundorte genannt worden.

N. thymi Wolff. Nach Fie ber nicht selten unter Thymus; ich fand ihn bei Sobieslau mehrmals unter Anthemis tinctoria auf einem Kalkhügel, auch in Gärten auf Mentha crispa und Melissa, noch Ende 9 in copula. Auch aus Jungfer-Teinitz, Chodau; Eger: am Ramberg, nicht zahlreich, 7 (D. T.)

N. senecionis Schill. An sandigen, unbebauten Orten und Schutthaufen, auf Anthemis, Erigeron, Senecio u. A. bei Prag (6-7) gemein, auch in Gärten auf verschiedenen PyrethrumArten. Blatna, Sobieslau, Chodau und gewiss auch in anderen Gegenden.

N. helveticus H.-Sch. (= obsoletus Fieb.) Bisher wenig beobachtet; im Frühjahre auf dem Weissen Berge bei Prag, unter trockenem Laub, bei Krumau (Anfang 9) unter Erdbeeren, Chodau (8).

N. punctipennis H.-Sch. An ähnlichen Orten wie die vorigen, unter Thymus, Potentilla, an Waldrändern unter abgefallenem Laube, nicht gemein; um Prag, Podiebrad, Hohenmauth, Sobieslau. 


\section{Cymus Hahn.}

C. glandicolor Hahn. An feuchten, sandigen Ufern, im Grase und unter verschiedenen Pflanzen; bei Sobieslau, selten (8), bei Neuhaus am 7. April an einem Waldrande auf Haidekraut gestreift; bei Eger: Königsberg, 7, selten (D. T.); auch aus Chodau (6-8).

C. claviculus Fall. An grasigen Waldblössen, unter Hypericum, Galium, Thymus, auch an sandigen Ufern unter Peplis, Illecebrum, Herniaria u. A., überall verbreitet; hie und da häufig; von 4-9, bei Sobieslau noch 9 in copula.

\section{Ischnorrhynchus Fieb.}

I. resedae Panz. (= didymus Zett.) In lichten Waldungen und an sonnigen Anhöhen, auf Birken, überall verbreitet, gewöhnlich mit Elasmostethus interstinctus, 5-8. Nach D. T. bei Eger (Kulm) auch auf Scabiosen.

b) var. flavicornis mihi. ${ }^{*}$ ) Mit der typischen Form auf Birken in der Umgebung von Prag, Jungfer-Teinitz, Chodau, ziemlich selten.

\section{Ischnodemus Fieb.}

I. sabuleti Fall. Lebt nach Fieber an feuchten Orten, an Flussufern auf Schilfrohr; nach einer schriftlichen Angabe des Dr. K. Sp ott bei Jungfer-Teinitz gefunden, doch habe ich das Insect in seiner Sammlung nicht gesehen.

\section{Geocoris Fall.}

G. grylloides Lin. An trockenen Feldrainen und sonnigen Hügeln, unter Thymus, Potentilla, Wachholder, um Prag selten; um Eger auf Stellaria und Scleranthus, bei St. Anna und Königswart zahlreich, auch bei Maria Kulm, 7 (D. T.).

*) Unter diesem Namen glaube ich eine charakteristische Abart unterscheiden zu dürfen, bei welcher Fühlerglied 1 rostgelb, 2 und $3 \mathrm{ganz}$ g e lb sind, und die schwarze Farbe der typischen Form sich höchstens auf eine ganz schmale Zeichnung der Basis des 2. Gliedes beschränkt. Durch diese Färbung der Fühlerglieder ist diese Abart dem I. geminatus Fieb. so ähnlich, dass ich sie nach der Beschreibung Fi eb er's und Put o n's für diese Art hielt und auch als solche in dem böhm. Verzeichnisse angeführt habe. Durch die Gefälligkeit des Hrn. Dr. G. v. Horváth erhielt ich echte I. geminatus, welche bedeutend kleiner sind, und an dem weiss beschuppten Vordertheile des Pronotum nur die zwei S-förmigen Querfurchen schwarz haben, wogegen bei der Varietät, sowie bei der typischen Form von $I$. resedae an dieser Stelle ein schwärzliches Querband zu sehen ist. 
G. dispar Waga (= Ullrichï Fieb.). Selten, mir bisher nur aus dem südlichem Böhmen bekannt; einmal fing ich diese Art bei Sobieslau auf Campanula glomerata (8), auch erhielt ich sie aus Tabor.

G. ater Fab. Mit der ersten Art an ähnlichen Orten, aber mehr verbreitet und auch häufiger (6-8).

G. siculus Fieb. Ein Exemplar dieser südlichen Art erhielt ich mit anderen Hemipteren aus Tabor (vom Hrn. Prof. Beranek); nach der Ansicht des Hrn. Dr. G. v. Horváth, welcher dieses Stück untersucht hatte, dürfte es vielleicht nur irrthümlich für eine heimische Art gehalten worden sein; denn es gehört der Stammform dieser Art an, welche vorwiegend in den Mittelmeerländern vorkommt. (Die ungarischen und südrussischen Exemplare bilden eine besondere Abart - var. arenarius Jak.)

\section{Camptotelus Fieb.}

C. lineolatus Schill. An sonnigen Anhöhen unter trockenem Moos und verschiedenen niedrigen Pflanzen, wahrscheinlich überall verbreitet, nach Fieber (W. B. p. 344) an manchen Orten häufig; bei Franzensbad unweit der Salzquelle zahlreich, 6 (D. T.).

\section{Microplax Fieb.}

M. interrupta Fieb. Mit dem Vorigen unter Thymus an sandigen Orten in der Umgebung von Prag (W. B. p. 315, 23), auch aus Pisek.

\section{Oxycarenus Fieb.}

0. modestus Fall. (= Spitzyi Fieb. W. B. T. II. Fig. 12.) Nach D. T. im Egerthal auf Erlen - 10.

O. Preyssleri Fieb. An sonnigen Hügeln unter Moos; Kuchelbad bei Prag, im September 1834 von Fieber zuerst entdeckt. (W. B. p. 313, 21).

\section{Plociomerus Say.}

P. fracticollis Schill. Unter Gras und verschiedenen Pflanzen an Teichdämmen und Torfwiesen, selten; Sobieslau; Franzensbader Moor, 7 gestreift (D. T.).

P. luridus H.-Sch. Von Hrn. Dr. v. Stein bei Chodau (5) gefunden.

\section{Plinthisus Westw.}

P. pusillus Scholtz. An sandigen Feldrainen unter Thymus, Potentilla, in Holzschlägen und trockenen Wäldern unter Moos 
172 L ad. D u da: Beiträge z. Kenntniss d. Hemipteren-Fauna Böhmens.

und Haidekraut, wohl überall verbreitet, an manchen Orten häufig, so z. B. bei Prag (Cibulka), Sobieslau, Blatna; bei Eger am Kammerbüchl auf Campanula, 7 (D. T.).

$P$. bidentulus H.-Sch. Selten, mir bisher nur von Podiebrad und Tabor bekannt; die brachyptere Form (P. brevipennis Latr.) fand ich im Frühjahre unter trockenem Laub am Weissen Berge bei Prag, auch bekam ich sie aus Leitomischl; Chodau (4).

\section{Lamprodema Fieb.}

L. maurum Fab. Unter Haidekraut und abgefallenem Laube in Wäldern und Holzschlägen, sehr selten; aus Blatna.

Pterotmetus Am. et Serv.

P. staphylinoides Burm. Mit der vorigen Art bei Blatna gefunden, mit vollkommen entwickelter Membran; wohl auch in anderen Gegenden verbreitet.

\section{Ischnocoris Fieb.}

I. angustulus Boh. (=hemipterus Stål, nec Schill.). Bei Sobieslau im Spätherbste unter Moos in Wäldern, nicht gemein, auch aus Blatna. Bei Eger am Bahndamme gegen Pograth, 6 (D. T.), ob wirklich diese Art oder der wahre I. hemipterus Schill?

I. punctulatus Fieb. Bei Sobieslau im Spätherbste unter Moos im Walde, selten.

\section{Rhyparochromus Curt. (Megalonotus Fieb.)}

$R$. chiragra Fab. Ueberall ziemlich gemein, auf Feldern, trockenen Triften und an Waldrändern zwischen Steinen, unter Moos und trockenem Laub; auch in Flussanschwemmungen (z. B. der Elbe) recht häufig.

R. praetextatus $\mathrm{H}$-Sch. Nach D. T. um Eger nicht selten: auf Wiesen und Blumen bei Königswart, Kammerbüchl und beim Viaduct, $6-7$.

$R$. antennatus Schill. Mit der ersten Art, vielleicht allgemein verbreitet, doch überall viel seltener.

R. hirsutus Fieb. Mit den vorigen, selten; von Prag, auch aus Blatna. 


\section{$2 \mathrm{BHL}$ Biodiversity Heritage Library}

Duda, Ladislav. 1885. "Beiträge zur Kenntniss der Hemipteren-Fauna Böhmens. 4. Fortsetzung." Wiener entomologische Zeitung 4, 169-172. https://doi.org/10.5962/bhl.part.20118.

View This Item Online: https://www.biodiversitylibrary.org/item/44101

DOI: https://doi.org/10.5962/bhl.part.20118

Permalink: https://www.biodiversitylibrary.org/partpdf/20118

\section{Holding Institution}

Smithsonian Libraries

\section{Sponsored by}

Smithsonian

\section{Copyright \& Reuse}

Copyright Status: NOT_IN_COPYRIGHT

This document was created from content at the Biodiversity Heritage Library, the world's largest open access digital library for biodiversity literature and archives. Visit BHL at https://www.biodiversitylibrary.org. 